\title{
Fast Multipole Method Applied to Volume Integral Equation Method
}

\author{
W. Hafla , A. Buchau, F. Groh, and W. M. Rucker \\ University of Stuttgart, Institute for Theory of Electrical Engineering, Pfaffenwaldring 47, 70569 Stuttgart, Germany
}

\begin{abstract}
The Volume Integral Equation Method (VIEM) has been used for the solution of three-dimensional nonlinear magnetostatic field problems. The number of unknowns is minimal as only the magnetic material has to be discretized. For accurate solutions of problems where the magnetic field is small compared to the excitation field a difference field formulation has been developed. To reduce computational costs the fast multipole method is applied both on compression of the system matrix and during post processing. The efficiency of the formulation is demonstrated in several examples.
\end{abstract}

\section{Introduction}

The Boundary Element Method (BEM) has already proved to be very applicable when linear magnetostatic problems are solved since only surface integrals have to be carried out. For problems with non-linear $B(H)$ characteristics additional volume integrals occur. This generalization of the BEM is called Volume Integral Equation Method (VIEM). Its number of unknowns is minimal since only magnetized materials have to be discretized. Also the surrounding space is taken into account inherently, contrary to the Finite Element Method for example.

The main drawback of the method is its fully dense system matrix. It implies that memory requirements and the number of operations to solve the system of equations with an iterative solver grow by $O\left(N^{2}\right)$, where $N$ is the number of unknowns. Also as integration is performed in three spatial directions, computational costs grow with third order with respect to the number of integration points.

A way to overcome these obstacles is to apply a suitable matrix compression technique. It can be used to compute the vector-by-matrix products during the iterative solving of linear system of equations. In Buchau et al. (2003a) it has been shown that with the fast multipole method (FMM) computational costs with BEM computations are reduced from $O\left(N^{2}\right)$ to approximately $O(N)$. As opposed to other compression techniques such as ACA (Buchau et al., 2003b)

Correspondence to: W. Hafla

(wolfgang.hafla@ite.uni-stuttgart.de) large reduction of computation time is also achieved with the FMM during post processing. This is why we decided to apply the FMM to the VIEM.

\section{Formulation}

The considered problem consists of free space, the magnetized material in the domain $\Omega_{m}$ with relative permeability $\mu_{r}$ and a solenoidal excitation field $\boldsymbol{H}_{S}$ due to free currents which can be computed with Biot-Savart's law. The total magnetic field can be split into the sum of the excitation field and the unknown induced field $\boldsymbol{H}_{i}$,

$\boldsymbol{H}(\boldsymbol{r})=\boldsymbol{H}_{S}(\boldsymbol{r})+\boldsymbol{H}_{i}(\boldsymbol{r})$.

If problems with highly permeably materials are solved $\boldsymbol{H}$ can become very small inside the magnetizable material compared to the excitation field. This is the case when $\boldsymbol{H}_{S}$ and $\boldsymbol{H}_{i}$ are almost anti-parallel. This is why huge cancellation errors usually occur. To overcome this problem a difference field formulation has been developed (Hafla et al., 2002) with which both linear and non-linear problems can be solved.

With Eq. (1) the basic equations for non-linear magnetostatic problems are

$\operatorname{rot}\left(\boldsymbol{H}_{S}+\boldsymbol{H}_{i}\right)=0$

$\operatorname{div} \boldsymbol{B}=0$

and the constitutive relation

$\boldsymbol{B}=\mu_{0} \boldsymbol{H}+\boldsymbol{M}=\mu_{0} \mu_{r}(|\boldsymbol{H}|) \boldsymbol{H}$

The induced field $\boldsymbol{H}_{i}$ can be represented by the integral formulation

$\boldsymbol{H}_{i}(\boldsymbol{r})=-\int_{V} \rho\left(\boldsymbol{r}^{\prime}\right) \nabla G\left(\boldsymbol{r}, \boldsymbol{r}^{\prime}\right) \mathrm{d} V^{\prime}-\int_{A} \sigma\left(\boldsymbol{r}^{\prime}\right) \nabla G\left(\boldsymbol{r}, \boldsymbol{r}^{\prime}\right) \mathrm{d} A^{\prime}(5)$

where $\sigma$ and $\rho$ are the equivalent surface and volume charges respectively and with Green's function of free space

$G\left(\boldsymbol{r}, \boldsymbol{r}^{\prime}\right)=\frac{1}{4 \pi \mu_{0}} \frac{1}{\left|\boldsymbol{r}-\boldsymbol{r}^{\prime}\right|}$. 
With this approach the irrotational condition Eq. (2) for $\boldsymbol{H}_{i}$ is always fulfilled. Considering induction's solenoidality Eq. (3) with Eq. (4) for points on the surface of $\Omega_{m}$ and within $\Omega_{m}$ gives two integral equations which have to be solved simultaneously (Babić et al., 2000; Kim et al., 2000).

\section{- Integral equation from induction's solenoidality on $\partial \Omega_{m}$}

Applying $\operatorname{div} \boldsymbol{B}=0$ on $\partial \Omega_{m}$ yields the integral equation (McWhirter et al., 1982)

$\frac{\sigma(\boldsymbol{r})}{2 \lambda(\boldsymbol{r})}-\left[\int_{A} \sigma\left(\boldsymbol{r}^{\prime}\right) \partial_{n} G\left(\boldsymbol{r}, \boldsymbol{r}^{\prime}\right) \mathrm{d} A^{\prime}+\int_{V} \rho\left(\boldsymbol{r}^{\prime}\right) \partial_{n} G\left(\boldsymbol{r}, \boldsymbol{r}^{\prime}\right) \mathrm{d} V^{\prime}\right]$

$=\boldsymbol{n}(\boldsymbol{r}) \cdot \boldsymbol{H}^{S}(\boldsymbol{r})$

with

$\partial_{n} G\left(\boldsymbol{r}, \boldsymbol{r}^{\prime}\right)=\boldsymbol{n}(\boldsymbol{r}) \cdot \nabla G\left(\boldsymbol{r}, \boldsymbol{r}^{\prime}\right)$,

$\lambda(\mathbf{r})=\frac{\mu_{r}(\boldsymbol{r})-1}{\mu_{r}(\boldsymbol{r})+1}$.

The normal vector $\boldsymbol{n}$ points outwards from $\Omega_{m}$. To avoid cancellation errors Eq. (7) can be solved in a first step for infinite permeability. In that case and if $\Omega_{m}$ is single-connected,

$\boldsymbol{H}=\left.\boldsymbol{H}^{i}\right|_{\mu_{r} \rightarrow \infty}+\boldsymbol{H}^{s}=0$,

so that Eq. (7) becomes an equation for the sources $\sigma_{\infty}$ of the scalar magnetic potential of the excitation field,

$$
\frac{1}{2} \sigma_{\infty}(\boldsymbol{r})+\int_{A} \sigma_{\infty}\left(\boldsymbol{r}^{\prime}\right) \partial_{n} G\left(\boldsymbol{r}, \boldsymbol{r}^{\prime}\right) \mathrm{d} A^{\prime}=\boldsymbol{n}(\boldsymbol{r}) \cdot \boldsymbol{H}_{S}(\boldsymbol{r})
$$

Once Eq. (11) has been solved, it can be used to substitute the right-hand side in Eq. (7). This leads with the difference surface charge

$\delta \sigma:=\sigma-\sigma_{\infty}$

to the integral equation

$$
\begin{aligned}
& \frac{\delta \sigma(\boldsymbol{r})}{2}+\lambda(\boldsymbol{r})\left[\int_{A} \delta \sigma\left(\boldsymbol{r}^{\prime}\right) \partial_{n} G\left(\boldsymbol{r}, \boldsymbol{r}^{\prime}\right) \mathrm{d} A^{\prime}+\int_{V} \rho\left(\boldsymbol{r}^{\prime}\right) \partial_{n} G\left(\boldsymbol{r}, \boldsymbol{r}^{\prime}\right) \mathrm{d} V^{\prime}\right] \\
& =\frac{\sigma_{\infty}(\boldsymbol{r})}{2}(\lambda(\boldsymbol{r})-1)
\end{aligned}
$$

From Eq. (10) follows

$$
\boldsymbol{H}^{S}(\boldsymbol{r})=-\left.\boldsymbol{H}^{i}(\boldsymbol{r})\right|_{\mu_{r} \rightarrow \infty}=\int_{A} \sigma_{\infty}\left(\boldsymbol{r}^{\prime}\right) \nabla G\left(\boldsymbol{r}, \boldsymbol{r}^{\prime}\right) \mathrm{d} A^{\prime}(14)
$$

so the induced magnetic field can be computed with

$$
\begin{aligned}
& \boldsymbol{H}(\boldsymbol{r})=-\int_{V} \rho\left(\boldsymbol{r}^{\prime}\right) \nabla G\left(\boldsymbol{r}, \boldsymbol{r}^{\prime}\right) \mathrm{d} V^{\prime} \\
& -\int_{A}\left\{\begin{array}{c}
\delta \sigma\left(\boldsymbol{r}^{\prime}\right) \\
{\left[\delta \sigma+\sigma_{\infty}\right]\left(\boldsymbol{r}^{\prime}\right)}
\end{array}\right\} \nabla G\left(\boldsymbol{r}, \boldsymbol{r}^{\prime}\right) \mathrm{d} A^{\prime}+\left\{\begin{array}{c}
0 \\
H_{S}(\boldsymbol{r})
\end{array}\right\} \\
& \text { if }\left\{\begin{array}{l}
\boldsymbol{r} \in \Omega_{m} \\
\boldsymbol{r} \notin \Omega_{m}
\end{array}\right\} \text {. }
\end{aligned}
$$

- Integral equation from induction's solenoidality in $\Omega_{m}$

In cases where the relative permeability $\mu_{r}(H)$ depends on the magnetic field, solenoidality of induction within $\Omega_{m}$ leads to non-vanishing volume charges (Krstajić et al., 1992)

$\rho(\boldsymbol{r})+\mu_{0} \boldsymbol{H}(\boldsymbol{r}) \cdot \nabla \ln \mu_{r}(\boldsymbol{r})=0$.

Substituting Eq. (15) into Eq. (16) for $\boldsymbol{r} \in \Omega_{m}$ gives

$\rho(\boldsymbol{r})-\int_{V} \rho\left(\boldsymbol{r}^{\prime}\right) \nabla G\left(\boldsymbol{r}, \boldsymbol{r}^{\prime}\right) \cdot \nabla \ln \mu_{r}(\boldsymbol{r}) \mathrm{d} V^{\prime}$
$-\int_{A} \delta \sigma\left(\boldsymbol{r}^{\prime}\right) \nabla G\left(\boldsymbol{r}, \boldsymbol{r}^{\prime}\right) \cdot \nabla \ln \mu_{r}(\boldsymbol{r}) \mathrm{d} A^{\prime}=0$.

\section{- Solution Procedure}

After Eq. (11) has been solved, the coupled system Eqs. (13), (17) can be solved. As it is in general non-linear an iterative procedure such as direct iteration has to be used where in every step Eq. (13), Eq. (17) is solved for a linear inhomogeneous distribution of $\mu_{r}$. Between every non-linear iteration step the distribution of relative permeability must be updated. To enable convergence for problems where $\mu_{r}(H)$ characteristics have a maximum, we use

$\mu_{r}^{(k)}=\left\{\begin{array}{c}B\left(H^{(k)}\right) /\left(\mu_{0} H^{(k)}\right), H^{(k)}<H_{m} \\ \tilde{B}^{(k)} /\left(\mu_{0} H\left(\tilde{B}^{(k)}\right)\right), H^{(k)} \geq H_{m}\end{array}\right.$

where $H_{m}$ is the field strength where this maximum occurs, $k$ denotes the iteration step number, and $H(B)$ is the inverse magnetization characteristic $H(B)$ with $\tilde{B}^{(k)}=\mu_{r}^{(k-1)} H^{(k)}$.

\section{Fast Multipole Method}

The fully dense system matrix of the IEM arises from the fact that all elements interact with all the other. In most cases the matrix itself is not explicitly needed but it is only used to compute matrix-by-vector products, as for iteratively solving the linear system of equations for example. In the FMM algorithm, the matrix-by-vector product can be split into a near-field part due to elements that are close to each other and a far-field part for the remaining elements,

$y=[A] \cdot\{x\}=\left[A_{\text {near }}\right] \cdot\{x\}+\left\{y_{\text {far }}\right\}$.

The division of element interactions into a near-field and a far-field part is carried out by means of a hierarchical grouping scheme that is based on cubes, the so-called octree. The near-field matrix $\left[A_{\text {near }}\right]$ is a sparse matrix that equals the total matrix with the far-field interactions removed. Elements whose distance from each other is sufficiently large are considered group-wise, where the size of the group depends on the distance. Their interactions are given respect to in $\left\{y_{f a r}\right\}$. Therefore Green's function is approximated by a truncated series expansion into spherical harmonics $Y_{n}^{-m}$. 

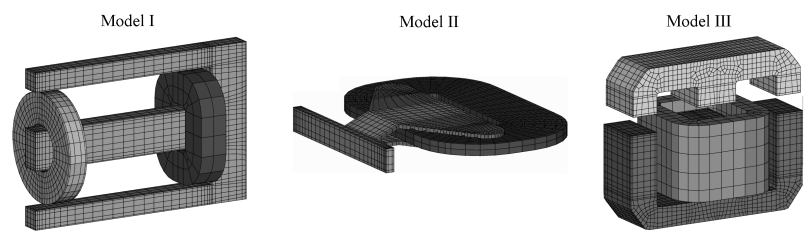

Fig. 1. Investigated models; finest meshes with problem oriented meshing strategy are shown.

The coefficients of the series expansion are called multipole coefficients $M_{n}^{m}$. For the presented formulations they can be calculated from the surface and volume charge densities,

$$
\begin{aligned}
& M_{n}^{m}=\int_{V} \rho\left(\boldsymbol{r}^{\prime}\right) \frac{1}{r^{\prime n+1}} Y_{n}^{-m}\left(\theta^{\prime}, \varphi^{\prime}\right) \mathrm{d} V^{\prime} \\
& +\int_{A} \sigma\left(\boldsymbol{r}^{\prime}\right) \frac{1}{r^{\prime n+1}} Y_{n}^{-m}\left(\theta^{\prime}, \varphi^{\prime}\right) \mathrm{d} A^{\prime} .
\end{aligned}
$$

We noticed that compared to BEM where only surface elements occur, it is much more important with the IEM to keep the number of near-field interactions as small as possible. This is because of two reasons. First, integrations with volume elements are in general much more time-consuming than with surface elements. Second, elements in the nearfield are often located in all three spatial directions and not only in two as in the case of BEM. The latter not only leads to more computation time but also to a lower memory compression rate. A small near-field requires higher order $L$ of series expansions. It was shown (Buchau et al., 2003a) that computational costs can be reduced with modified transformations of the multipole coefficients from $O\left(L^{4}\right)$ to $O\left(L^{3}\right)$.

Also, the different sizes of surface and volume elements have to be considered for the grouping scheme (Buchau et al., 2003c).

\section{Numerical Results}

The coupled system of Eqs. (13) and (17) has been discretized with Galerkin's method and second order isoparamteric volume and surface nodal elements. GMRES with Jacobi preconditioner was used as linear solver. In the following the properties of the FMM with respect to the IEM are investigated. Then an example field problem is presented.

\subsection{Fast Multipole Method}

We investigated the application of the FMM to the IEM concerning three aspects that may influence compression rates. The first is the overall shape of the model. The second is the influence of the two different meshing strategies, problem oriented meshing and meshing with the aim to create as many as possible elements of the same size. And third, how the compression rate depends on the number of degrees of freedom (DOF).
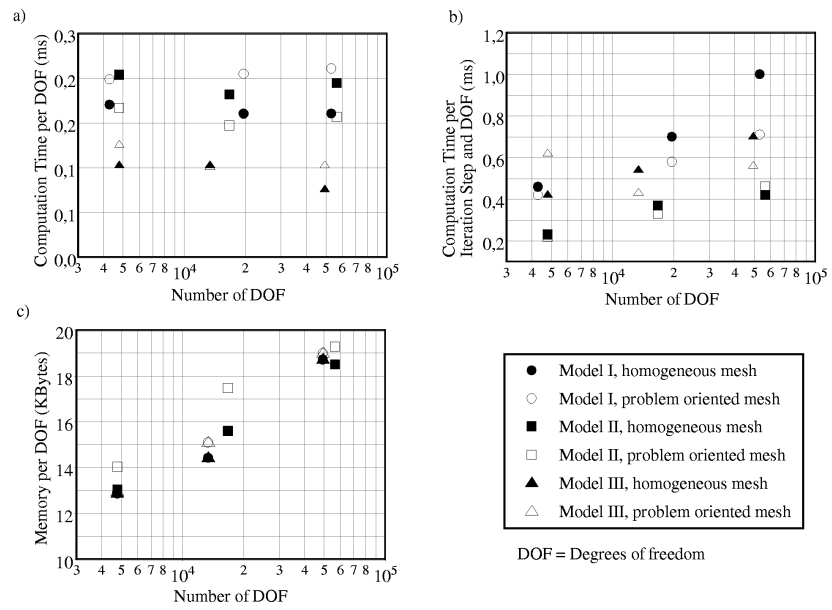

Fig. 2. Computational costs by application of the FMM.

The three different models shown in Fig. 1 were used for these investigations. Therefore all of them where meshed with both problem oriented and homogeneous meshes. Again for each of these two cases three different meshes with different number of elements where generated, so all in all 18 different meshes were used.

\section{- Overall Model Shape}

With BEM near-field interactions between surface elements are often found only in two dimensions. With volume elements near-interactions can occur up to all three dimensions depending on the overall model structure. Therefore we used the models in Fig. 1 with long and thin structures (Model I), flat structures (Model II) and bellied structures (Model III).

As it can be seen from Fig. 2a where the time per DOF for near-field assembling is shown, the time for model III is the lowest while the two other models are nearly the same. The time per DOF and iteration step during solving of the linear system of equations is displayed in Fig. 2b. In Fig. 2c memory requirements per DOF are shown. They depend roughly only on the number of DOF and not on the respective model.

\section{- Meshing Strategy}

When the FMM is applied to BEM calculations it becomes apparent that mesh homogeneity can have a significant influence on the compression rate. In conventional BEM one usually tries to use as few elements as possible. This mostly results in meshes with elements of largely varying sizes. The geometrical dimensions of the near-field of elements of large size are usually large. This means that if many small elements lie in the neighborhood of large elements, a high number of near-field integrations have to be computed which results in a lower compression rate. To investigate this we considered both homogeneous and problem oriented meshes. 

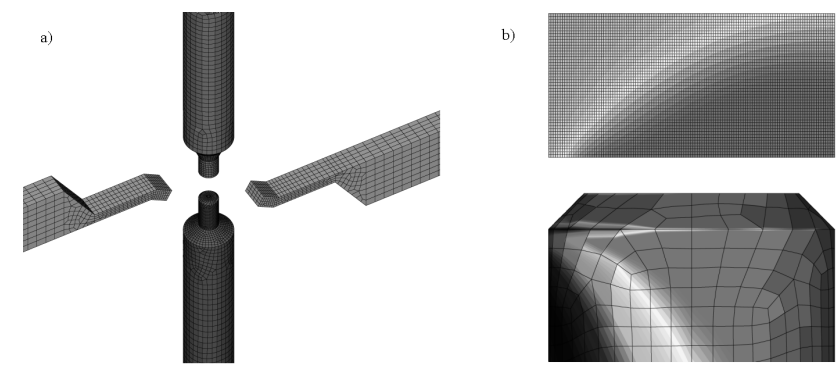

Fig. 3. Investigated arrangement of pole shoes for Magnetic Transmission X-ray Microscopy Project (6812 surface elements, 3130 volume elements, 36912 unknowns).

From Figs. $2 \mathrm{a}$ and $2 \mathrm{c}$ it can be seen that the increased size of the near-field of problem oriented meshes is reflected in increased computational costs for near-field assembly as well as an increase of total memory requirements.

\section{- Number of Degrees of Freedom}

For all three models different mesh sizes where used, both for homogeneous and problem oriented meshes. The assembly time per DOF of the near-field matrix in Fig. 2a is approximately independent of the number of DOF. Except for the coarse problem oriented mesh a logarithmic dependency between the number of DOF and computation time can be seen in Fig. 2b. The increase of memory requirements per DOF in Fig. 2c is believed to be caused by a growth of average near-field interactions due to the increasing number of volume elements.

\subsection{Difference Field Formulation}

For the Magnetic Transmission X-ray Microscopy Project at BESSY II (Eimüller, 2002) the field distribution in the air region between the pole shoes shown in Fig. 3a was investigated.

The flux lines run between the horizontal pole shoes. The influence of the passive vertical pole shoes was to be investigated especially at the sample location which is directly above the lower one. The vertical pole shoes don't saturate, so their relative permeability could be assumed constant, e.g. 1700 even for high accuracy calculation.

The stopping criterion of mean $(\Delta \mu / \mu)<2.5 \%$ of the nonlinear solver was chosen to obtain accurate results in the region of interest. It was reached after 28 iterations and about $110 \mathrm{~h}$. As the system matrix was compressed with the FMM only 729 MByte were required to solve the problem. Without the FMM about 10.1 GByte would have been required. This equals a compression rate of $93 \%$.

Flux density was calculated at the nodes of the very fine evaluation mesh (19101 nodes) shown in Fig. 3b. The calculation of the field at all 28389 evaluation points took only 2 min.

\section{Conclusions}

A new indirect Volume Integral Equation Method formulation for non-linear magnetostatic problems was developed. Uniform treatment of surface and volume charges results in an elegant coupling of integral equations. Non-linear problems are tackled by consecutively solving linear problems. It has been shown that application of the fast multipole method leads to a large reduction of computational costs and therefore to an efficient and practicable alternative to e.g. Finite Element Method.

Acknowledgements. We would like to thank J. Konarev for the creation of discretized models and for the thorough compilation of the obtained results.

\section{References}

Babic, S., Akyel, C., and Gavrilovic, M.: Calculation improvement of $3 \mathrm{~d}$ linear magnetostatic field based on fictious magnetic surface charge, IEEE Trans. Magn., vol. 36, no. 5, 3125-3127, Sept. 2000.

Buchau, A., Hafla, W., and Rucker, W. M.: Fast and efficient 3d boundary element method for closed domains, Symposium Reports of the Sixth International Symposium on Electric and Magnetic Fields, 211-214, Aachen, 2003a.

Buchau, A., Rucker, W. M., Rain, O., Rischmüller, V., Kurz, S., and Rjasanow, S.: Comparison between different approaches for fast and efficient 3d BEM computations, IEEE Trans. Magn., vol. 39, no. 3, 1107-1110, 2003b.

Buchau, A., Hafla, W., Groh, F., and Rucker, W. M.: Grouping schemes and meshing strategies for the fast multipole method, COMPEL, vol. 22, no. 3, 495-507, 2003c.

Eimüller, T.: Magnetic imaging of nanostructured systems with Transmission X-ray Microscopy, Dissertation, Universität Würzburg, 2002.

Hafla, W., Groh, F., Buchau, A., and Rucker, W. M.: Magnetostatic field computations by an integral equation method using a difference field concept and the fast multipole method, Proceedings of the 10th IGTE Symposium on Numerical Field Calculation in Electrical Engineering, 262-266, 2002.

Kim, D., Park, I., and Park, M., and Lee, H.: 3-d magnetostatic field calculation by a single layer boundary integral equation method using a difference field concept, IEEE Trans. Magn., vol. 36, no. 5, 3134-3136, Sept. 2000.

Krstajić, B., Andelić, Z., Milojković, S., and Babić, S.: Nonlinear $3 \mathrm{~d}$ magnetostatic field calculation by the integral equation method with surface and volume magnetic charges, IEEE Trans. Magn., vol. 28, no. 2, 1088-1091, March 1992.

McWhirter, J. H., Oravec, J. J., and Haak, R. W.: Computation of magnetostatic fields in three-dimensions based on Fredholm integral equations, IEEE Trans. Magn., vol. MAG-18, no. 2, 456460, March 1982. 